\section{Vascular basis of late-onset depressive disorder ${ }^{\dagger}$}

\author{
ROBERT C. BALDWIN and JOHN O'BRIEN
}

Arguably, in later adult life depressive disorder is a more heterogeneous condition than when it occurs earlier on. A broad distinction is often made between early-onset and late-onset depression, but there remains disagreement as to precisely what age should divide the two. Fifty years of age is the most widely used cut-off, particularly in the USA, although in the UK 65 years is often chosen. Although inevitably arbitrary, the choice of 50 years does tally with what is known about the familial risk in affective disorder, the risk being much reduced from this age onwards (Caine et al, 1994). As many as three-quarters of patients with major depressive disorder who present to old age psychiatrists have a late-onset form of the illness, and this is associated with considerable morbidity, increased mortality and treatment resistance. Late-onset depressive disorder has been the subject of recent research which suggests that it is associated with specific aetiological, clinical and neurobiological factors and, particularly, that it has a close and important relationship with underlying cerebrovascular disease. This paper summarises recent literature addressing this issue and examines implications for clinical practice and research.

\section{CLINICAL ASPECTS}

Although some symptoms such as delusions, hypochondriasis and anxiety are reportedly more common in late-onset depression, the evidence is inconsistent (summarised by Caine et al, 1994) and certainly less robust than the data cited above pertaining to genetic risk. The suggestion is that early-onset depression is associated with an increased genetic risk along with a cognitive vulnerability to depression, but that vulnerability to late-onset

dysfunction, apathy and psychomotor

change. This has implications for both treatment and prevention.

Declaration of interest None. depression may occur by other internal mechanisms and be triggered by adverse events which of themselves would not ordinarily cause depression. That this vulnerability may be due to changes in the brain itself is supported by findings of increased cognitive dysfunction in patients with late-onset versus early-onset depression (Salloway et al, 1996) and an increased rate of brain abnormalities.

\section{Relationship between depression and cardiovascular disease}

It is now clear that there is a strong relationship between depression and vascular disease, at least in the form of cardiovascular disease, although this association is not limited to the elderly. A number of epidemiological studies of younger subjects have reported a prior history of depression to be associated with the subsequent development of ischaemic heart disease (reviewed by Glassman \& Shapiro, 1998). This association remains after controlling for potential confounders such as family history, blood pressure, smoking, obesity and levels of physical activity. These findings are important in confirming the wellrecognised observation from hospital-based studies of increased mortality, particularly from cardiovascular and cerebrovascular deaths, in elderly patients with depression. In addition to people with depression being at increased risk of cardiovascular disease, the presence of depression in those with established cardiac disease confers a poor prognosis in terms of cardiac mortality. The classic 18-month follow-up study by Frasure-Smith et al (1995) controlled for potential confounders and found that the influence of depression on mortality (odds ratio 3.6) was approximately the same as that of the strongest other predictor of mortality after myocardial infarct, heart failure.

However, the nature of this association is unclear. One possibility is that depression directly causes cardiovascular disease. Potential mechanisms could include an increased thrombotic tendency in people with this condition, since depression has been associated with factors that increase platelet aggregation (Laghrissi-Thode et al, 1997), and the effects of hypercortisolaemia or of immune activation, which has also been demonstrated in depression. Shared genetic risk factors may be another possibility. The apolipoprotein $\varepsilon 4$ allele is an established risk factor for coronary artery disease (as well as for 
Alzheimer's disease), and although reports of a possible increased frequency of the $\varepsilon 4$ allele in late-life depression are inconsistent, other shared genetic factors may exist. Another possibility is that both depression and ischaemic heart disease are manifestations of a common pathological process, the most obvious candidate being atherosclerosis. This would support the idea that atherosclerotic disease of the brain predisposes to depression. Of course, these possibilities are not mutually exclusive, and it may be that the presence of vascular disease may predispose to depression, which may itself further exacerbate the process.

\section{Relationship between depression and cerebrovascular disease}

More direct evidence that vascular disease of the brain may predispose to depression in late life comes from several studies suggesting a strong link between depression and cerebrovascular disease. First, it is well established that there is a high prevalence of depression in vascular dementia compared with Alzheimer's disease (Newman, 1999). Second, stroke can lead to depression. This has been estimated to affect between $20 \%$ and $50 \%$ of patients in the first year after stroke (House et al, 1991). Lesions located close to the anterior pole of the left hemisphere reportedly show the greatest association with depression (Robinson et al, 1984), although this relationship has been questioned by some (MacHale et al, 1998). Third, patients with cerebrovascular disease in the absence of depression often develop lability of mood (emotionalism). Fourth, an unexpectedly high rate of silent stroke has also been reported in people with depression, the presence of which may increase vulnerability to depression, particularly in the absence of genetic predisposition or significant psychosocial precipitants (Fujikawa $e t$ al, 1997). Finally, in the absence of overt evidence of stroke, recent research has emphasised an association of subtle abnormalities of white matter and deep grey matter, of possible vascular origin, with late-onset depression both in hospital patients (O'Brien et al, 1996; Greenwald et al, 1998) and people living in the community (Steffens et al, 1999). The hyperintensities, best visualised on magnetic resonance imaging (MRI) using dual echo (proton density and $T_{2}$-weighted) or fluid-attenuated inversion recovery (FLAIR) sequences, are predominantly found in frontal lobes and basal ganglia (Greenwald et al, 1998).

These various lines of evidence have led to the proposition that vascular brain disease is an important cause of late-onset depression, termed 'vascular depression' (Alexopoulos et al, 1997; Krishnan et al, 1997). It is known that diseases affecting the subcortex are associated with a high rate of depression and so it is a reasonable hypothesis that vascular damage to frontosubcortical structures is an important aetiological factor in late-life depression. Damage to end-arteries supplying subcortical striato-pallido-thalamo-cortical pathways may disrupt neurotransmitter circuitry involved in mood regulation, thus causing or predisposing to depression. This may potentially occur either via strategically placed lesions or as a result of an overall burden or threshold effect.

Apart from the evidence cited above, support for the notion of vascular depression comes from clinical differences that may exist between vascular and non-vascular cases. Cases of vascular depression have been associated with late-onset depression (O'Brien et al, 1996; Alexopoulos et al, 1997; Krishnan et al, 1997); an increase in vascular risk factors (Baldwin \& Tomenson, 1995); particular symptoms - reduced depressive ideation and increased psychomotor retardation (Alexopoulos et al, 1997); and greater cognitive impairment, especially executive dysfunction (Alexopoulos et al, 1997). People with vascular depression, defined by the presence of hyperintensities on MRI, have a poorer response to antidepressant treatment than people with non-vascular depression (Simpson et al, 1998). The last study also demonstrated that lesion location more than severity had predictive value - frontal white-matter hyperintensity, and pontine and basal ganglia lesions predicted poorer outcome - and that the presence of extrapyramidal signs, impaired motor sequencing and grasp reflex were associated with both subcortical lesions and poor outcome, suggesting that neurological impairment may be a good marker for the presence of white-matter (and other) lesions seen on MRI. Other studies have shown that at baseline the amount of white-matter hyperintensity predicts poorer longer-term recovery, relapse (O'Brien et al, 1998) and possibly the later development of vascular dementia (Hickie et al, 1997).

\section{DIAGNOSTIC ISSUES}

There is currently no consensus on what criteria should be used for making a diagnosis of vascular depression, although defining features have been proposed (Steffens \& Krishnan, 1998). These included the presence of vascular risk factors and/or hyperintensities on MRI. However, variation in assessing and measuring scan findings means that, as with vascular dementia, clearly defined criteria with good reliability between centres may be difficult to achieve. Importantly, unlike post-stroke depression or vascular dementia, a diagnosis of vascular depression does not (and is unlikely to) require a causal or temporal relationship between the mood disorder and cerebrovascular changes within the brain, although there is tentative evidence that the direction of causality is such that cerebrovascular risk is predictive of later depression (Lyness et al, 2000). As it happens, history has come full circle. Gaupp in 1905 delineated a subgroup of 45 elderly patients (out of 351 first referrals) with 'arteriosclerotic depression' (quoted by Post, 1962). While further work will determine the clinical usefulness of the concept of vascular depression, it would seem prudent to use the term when there is clear evidence of neuroimaging changes consistent with the diagnosis, rather than simply to indicate the presence of vascular risk factors in patients with depression.

\section{CRITIQUE OF THE CONCEPT OF VASCULAR DEPRESSION}

Despite the arguments presented above, there is currently little hard scientific evidence to support the vascular depression hypothesis. The studies cited are associative rather than causal, and it is still possible that unrecognised confounders explain the associations between depression and vascular disease and between depression and cerebral hyperintensities on brain imaging. Although assumed to be vascular, the pathological basis of the hyperintensities in people with depression has not been determined. Some authors report that if patients with depression who also have vascular disease are excluded from study, white-matter hyperintensities are no more common in participants with depression alone than in healthy control subjects (Miller et al, 1994), although others disagree and find such hyperintensities to 
be associated with depression even after accounting for known vascular risk factors (O’Brien et al, 1996). Similar lesions in other subgroups, including those with dementia and people without dementia who are dying of other causes, have been examined and reveal a diverse pathological basis which does not always include atherosclerotic disease. While atherosclerosis remains the most likely explanation of such changes, clear demonstration that this is the case is still required. Vascular depression implies structural neuropathological changes in brain areas implicated in mood disturbance, such as the basal ganglia and frontal lobes, although reliable neuropathological evidence for this is lacking at present. Loss of glial cells and reductions in cortical thickness and neuronal size in the frontal cortex of people with depression have been reported (Rajkowska et al, 1999), but their cause remains unclear. A report of increased expression of intracellular adhesion molecule 1 (ICAM-1) in the dorsolateral prefrontal cortex of elderly people with depression compared with a similarly aged group without depression (Thomas et al, 2000) is of interest because this marker is upregulated in the presence of cerebral hypoxia, which may be consequent on atheromatous changes in vessels. However, further investigation is required before such studies can be taken as clear support for the vascular depression hypothesis.

\section{IMPLICATIONS FOR PRACTICE}

The knowledge of a close relationship between depression in the elderly and both cardiovascular and cerebrovascular disease should, at the very least, prompt careful assessment and appropriate management of both. This may involve antidepressant treatment, antiplatelet therapy, cardiovascular medication and other therapeutic avenues. These approaches imply close collaboration between psychiatrists and others, including geriatricians, cardiologists and neuroradiologists. The principles of treating patients with 'vascular depression' do not differ from other cases. However, the possibility of associated cardiovascular abnormality will influence the choice of antidepressant. Also, there is some evidence that response to pharmacological therapy is somewhat less than that to electroconvulsive therapy (Simpson et al, 1998),

\section{CLINICAL IMPLICATIONS}

- There is growing evidence for a subtype of depression often - although not always - occurring in later life. It may have its origins in cerebrovascular disease and has been termed 'vascular depression'.

- Vascular depression may be diagnosed from a combination of reduced depressive ideation, greater psychomotor disturbance, apathy, executive dysfunction on neuropsychological testing, and neuroimaging abnormalities in the basal ganglia and white matter on magnetic resonance imaging (MRI).

- Vascular depression has important implications for understanding the pathogenesis, treatment and prevention of depressive disorder.

\section{LIMITATIONS}

Much of the evidence supporting the concept of vascular depression is associative rather than causal.

- Studies of white-matter and other abnormalities seen on MRI have shown that the pathological change is heterogeneous and not confined to vascular disease. It is certainly not unique to depressive disorder in older people.

- The relationship between vascular depression and dementia is an important, but as yet unclear, issue.

ROBERT C. BALDWIN, FRCPsych, Manchester Royal Infirmary, Manchester; JOHN O'BRIEN, MRCPsych, Wolfson Research Centre, Institute for the Health of the Elderly, Newcastle General Hospital, Newcastle upon Tyne, UK

Correspondence: Dr Robert C. Baldwin, Consultant in Old Age Psychiatry, York House, Manchester Royal Infirmary, Oxford Road, Manchester MI3 9BX, UK. Tel: +44 (0) I6I 276 53I7; Fax: +44 (0) I6I 276 5303; e-mail: Robert.Baldwin@man.ac.uk

(First received 6 June 2000, final revision 2 May 200I, accepted 23 May 200I)

although the latter is more likely to provoke delirium in patients who have extensive white-matter hyperintensities and other changes in the brain (Figiel et al, 1990). The concept of vascular depression may also lead to non-pharmacological strategies aimed at treating amotivational syndromes in depression that arise from subcortical brain damage, and to appropriate psychoeducation and support of carers who have to manage the patient with brain-based apathy.

However, the vascular depression hypothesis, if substantiated through further research, potentially has much wider implications. It suggests the possibility of new therapeutic interventions more akin to the management of cardiac disease and vascular dementia. It might allow the early identification of those at subsequent risk of late-onset depression, allowing new strategies aimed as much at prevention as at treatment. Demonstration that damage to key brain areas is important in the development of depression would encourage detailed neuroanatomical and neuropathological study of these areas, establishing their role in affective and cognitive dysfunction. This would inform research into the causes and anatomical substrates of mood disturbance in 'non-vascular depression', opening new avenues of study which might lead to better treatments and outcomes for depression in people of all ages. In the meantime, knowledge that hyperintensities on MRI predict treatment resistance and poor outcome should lead to vigorous treatment and careful follow-up of such cases in clinical practice. 


\section{REFERENCES}

Alexopoulos, G. S., Meyers, B. S., Young, R. C., et a (1997) Clinically defined vascular depression. American journal of Psychiatry, 154, 562-565.

Baldwin, R. C. \& Tomenson, B. (1995) Depression in later life. A comparison of symptoms and risk factors in early and late onset cases. British Journal of Psychiatry, 167, 649-652.

Caine, E. D., Lyness, J. M., King, D. A., et al (1994) Clinical and etiological heterogeneity of mood disorders in elderly patients. In Diagnosis and Treatment of Depression in Late Life: Results of the NIH Consensus Development Conference (eds L. S. Schneider, C. F. Reynolds, B. D. Lebowitz, et al), pp. 23-53. Washington, DC: American Psychiatric Press.

Figiel, G. S., Coffey, C. E., Djang, W. T., et al (1990) Brain magnetic resonance imaging findings in ECTinduced delirium. Journal of Neuropsychiatry and Clinical Neurosciences, 2, 53-58.

Frasure-Smith, N., Lesperance, F. \& Talajic, M. (1995) Depression and 18-month prognosis after myocardial infarction. Circulation, 91, 999-1005.

Fujikawa, T., Yanai, I. \& Yamawaki, S. (1997)

Psychosocial stressors in patients with major depression and silent cerebral infarction. Stroke, 28, |123-1125.

Glassman, A. H. \& Shapiro, P. A. (1998) Depression and the course of coronary artery disease. American Journal of Psychiatry, 155, 4-1I.

Greenwald, B. S., Kramer-Ginsberg, E., Krishnan, K. R. R., et al (1998) Neuroanatomic localization of magnetic resonance imaging signal hyperintensities in geriatric depression. Stroke, 29, 613-617.

Hickie, I., Scott, E., Wilhelm, K., et al (1997)

Subcortical hyperintensities on magnetic resonance imaging in patients with severe depression: a longitudinal evaluation. Biological Psychiatry, 42, 367-374.

House, A., Dennis, M., Mogridge, L., et al (1991) Mood disorders in the year after first stroke. British Journal of Psychiatry, 158, 83-92.

Krishnan, K. R., Hays, J. C. \& Blazer, D. G. (1997) MRI-defined vascular depression. American journal of Psychiatry, I54, 497-50I.

Laghrissi-Thode, F., Wagner,W. R., Pollock, B. G., et a (1997) Elevated platelet factor 4 and beta-

thromboglobulin plasma levels in depressed patients with ischemic heart disease. Biological Psychiatry, 42 290-295.

Lyness, J. M., King, D. A., Conwell, Y., et al (2000) Cerebrovascular risk factors and I-year depression outcome in older primary care patients. American Journal of Psychiatry, I57, 1499-1501.

MacHale, S. M., O'Rourke, S. J., Wardlaw, J. M., et a (1998) Depression and its relation to lesion location after stroke. Journal of Neurology, Neurosurgery and Psychiatry, 64, 37I-374.

Miller, D. S., Kumar, A., Yousem, D. M., et al (1994) MRI high-intensity signals in late-life depression and Alzheimer's disease. American Journal of Geriatric Psychiatry, 2, 332-337.

Newman, S. C. (1999) The prevalence of depression in Alzheimer's disease and vascular dementia in a population sample. Journal of Affective Disorders, 52, 169-176.

O'Brien, J., Desmond, P., Ames, D., et al (1996) A magnetic resonance imaging study of white matter lesions in depression and Alzheimer's disease. British Journal of Psychiatry, 168, 477-485.

_ , Ames, D., Chiu, E., et al (1998) Severe deep white matter lesions and outcome in elderly patients with major depressive disorder: a follow-up study. BMJ, 317 982-984.

Post, F. (1962) The Significance of Affective Symptoms in Old Age. Institute of Psychiatry Maudsley Monographs 10, p. 4. Oxford: Oxford University Press.

Rajkowska, G., Miguel-Hidalgo, J. J., Wei, J., et al (1999) Morphometric evidence for neuronal and glial prefrontal cell pathology in major depression. Biologica Psychiatry, 45, 1085-1098.

Robinson, R. G., Kubos, K. L., Starr, L. K., et al (1984) Mood disorders in stroke patients: importance of location of the lesion. Brain, 107, 81-93.

Salloway, S., Malloy, P., Kohn, R., et al (1996) MR and neuropsychological differences in early- and latelife-onset geriatric depression. Neurology, 46 , $567-1574$

Simpson, S., Baldwin, R. C., Jackson, A., et al (1998) Is subcortical disease associated with a poor response to antidepressant treatment? Neurological, neuropsychological and neuroimaging findings. Psychological Medicine, 28, I0I5-1026.

Steffens, D. C. \& Krishnan, K. R. (1998) Structural neuroimaging and mood disorders: recent findings, implications for classification, and future directions. Biological Psychiatry, 43, 705-712.

\section{_ , Helms, M. J., Krishnan, K. R., et al (1999)}

Cerebrovascular disease and depression symptoms in the cardiovascular health study. Stroke, 30. 2159-2166

Thomas, A. J., Ferrier, I. N., Kalaria, R. N., et a (2000) Expression of ICAM-I is increased in the dorsolateral prefrontal cortex in late-life depression. American Journal of Psychiatry, 157, 1682-1684. 